Y. C. Minh and E. F. van Dishoeck, eds.

\title{
After Dinner Talk: From Molecular Clouds to Life on Various Planetary Systems
}

\author{
Norio Kaifu \\ Subaru Telescope, National Astronomical Observatory of Japan, 650 \\ North Aohoku Place, Hilo, HI 96720, USA
} aloha!

An-nyong ha-seyo, good evening ladies and gentlemen, minasan konbanwa,

We have enjoyed a pleasant dinner and the beauty in Korea during the symposium. I would like to express my deep thanks to Dr. Ewine van Dishoeck, Dr. Young Chol Minh, and all the organizers for their tremendous efforts preparing such an exciting symposium and for letting me have the honor to speak here.

One of the most impressive aspects in this symposium is the very wide range of activities in the investigations of interstellar matter involving observations in various wavelength regions toward various kinds of objects such as stars, clouds, young stars, proto-planetary disks and the solar system. At this opportunity, I would like to talk about the astronomical observations toward "life in the universe," which has been my long-term keyword. As Dr. Ohishi kindly introduced me, I have been studying interstellar matter and star formation by means of mmwave and IR observations for 30 years. On the other hand, I also have spent much of my time with the development and construction of new telescopes and new observing instruments. Therefore, my perspective is mainly as an observer.

Let me start this talk with the history of interstellar molecules, mentioning some of my own experiences, and then we will turn our heads back to look towards the future. As this is a dinner talk, I will try my best to talk for both guests and my professional colleagues.

\section{Snyder and Buhl's Dream}

In the kick-off lecture on Monday, Professor Dalgarno started his review of astrochemistry in 1929, by mentioning Eddington's pessimistic prediction. In those days, the universe was believed to be completely dry and vacant but filled with dangerous ultraviolet radiation, making it impossible for life anywhere except here on earth. My own history of astrochemistry started in 1968, when the group of Professor Charles Townes found radio spectra of ammonia and water vapor molecules in interstellar clouds using their small radio telescope. Charles Townes made an extensive review of the possible molecules in the universe in 1957 and the above-mentioned detection was the fruit of his continued efforts to observe such molecules. This discovery was very surprising at that time, but the detection of $\mathrm{H}_{2} \mathrm{CO}$ (formaldehyde) by Lewis Snyder and David Buhl immedi- 
ately following it was almost a sensation, because it is a molecule containing two heavy atoms, oxygen and carbon. More importantly, it is an organic molecule!

I remember an article by L. Snyder and D. Buhl entitled "From Radio Astronomy towards Astrochemistry," which appeared in the April 1971 issue of the Technology Review magazine. They mentioned at the end of their article: "Thus we are learning something about the possible seeds of life existing in clouds out of which planets are being formed in other parts of our galaxy, and we are also discovering what conditions may have been like in our own solar nebulae five billion years ago". Probably for many readers, Snyder and Buhl's perspective expressed here might be felt much too premature. However, I was strongly impressed by their ambition.

At that time, I was a graduate student involved in a small group of radio astronomers lead by Kenji Akabane and Masaki Morimoto at the Tokyo Astronomical Observatory, and the construction of a 6-m diameter small mm-wave telescope had just been started at Mitaka. I immediately visited Professor Koichi Shimoda, a world famous molecular physicist, to discuss the possibility of detecting $\mathrm{mm}$-wave rotational spectra of various molecules which may exist in the interstellar matter. Morimoto and myself started to build a radio spectrometer to detect $\mathrm{mm}$-wave spectra of molecules which might exist in interstellar space. We focused on the detection of para- $\mathrm{H}_{2} \mathrm{CO}, \mathrm{HCN}$ and $\mathrm{CO}$. The "gold-rush" of detection of many interstellar molecules happened in the United States, especially at Kitt Peak, in Arizona. This was still a very exciting time for us in Japan too, with the opening of a new field of science.

I show here a result of a frequency scan survey of molecular lines toward a dark cloud in Taurus. This was a long-term project using the Nobeyama 45- $\mathrm{m} \mathrm{mm}$-wave telescope, which we completed in 1982 following the initial 6$\mathrm{m} \mathrm{mm}$-wave telescope at Mitaka. Dark clouds are fundamentally important as the source material of stars, planets, and consequently, of life. Such surveys are important because we might be able to detect molecules which we cannot predict from existing knowledge. We throw a big net towards the dark clouds and pick up new spectral lines which we cannot identify with any line of known molecule. Such surveys have never been performed towards dark clouds before, because dark clouds are cold and naturally the molecular lines are weak and narrow. It took us more than 10 years to obtain 2-million spectral data though we were using the high sensitivity 45-m telescope and super-large 32,000 channel acousto-optical radio spectrometer. As a result, we caught 10 unknown fishesall exotic organic molecules never known on earth before. Dark clouds are now known to be a rich source of chemical reactions to form $\mathrm{H}_{2} \mathrm{O}, \mathrm{NH}_{3}, \mathrm{H}_{2} \mathrm{CO}, \mathrm{HCN}$, various carbon chains and numerous other organic compounds.

\section{Progress of Observations}

The progress of observing interstellar matter with a number of new mm-wave telescopes, IR telescopes and HST etc. is magnificent. In the early period of detections of interstellar molecules, we knew almost nothing about the formation processes of stars and planets. At this symposium, we are discussing the detailed process of planetary system formation comparing tremendous observational data with a variety of theoretical and experimental results. We are talking about the 


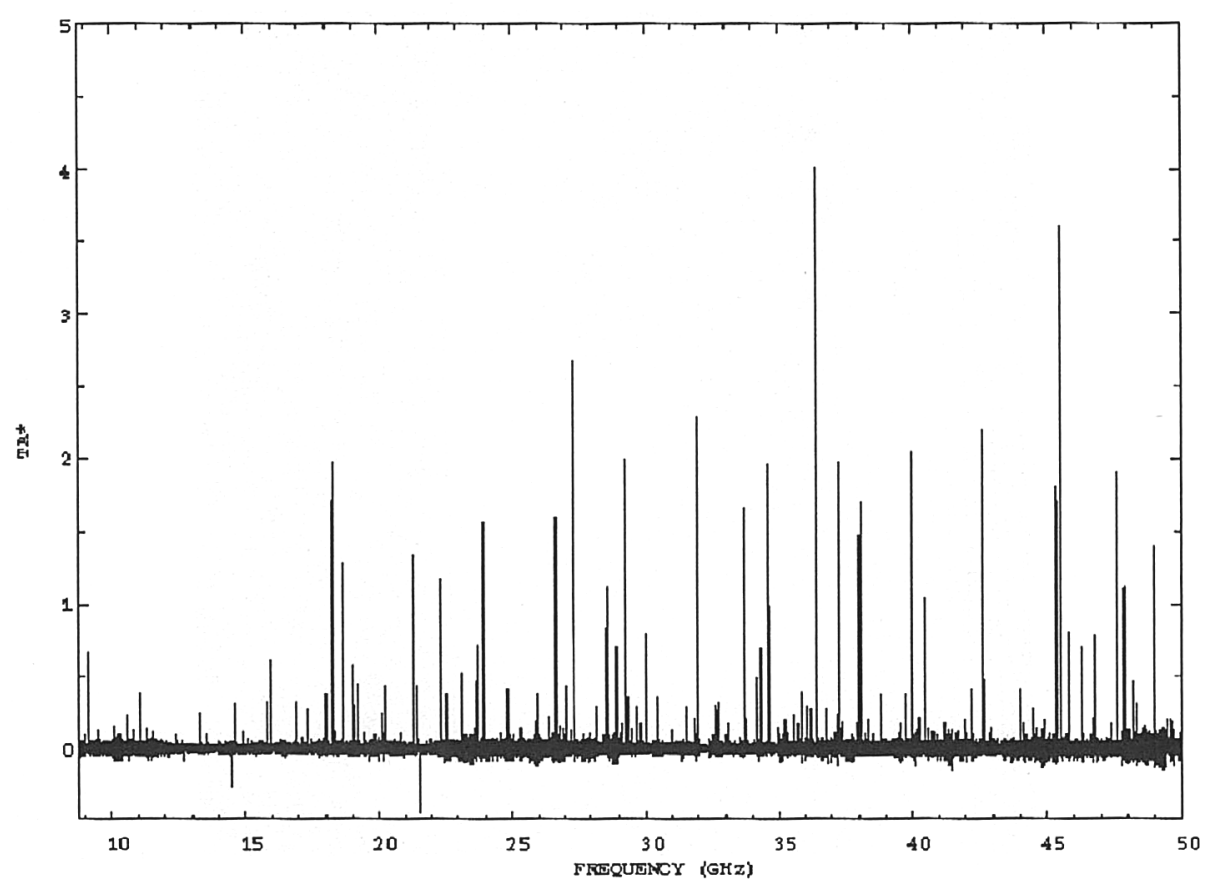

Figure 1. A millimeter-wave frequency scan of molecular lines toward the dark cloud TMC-1, obtained with the Nobeyama 45-m telescope and acousto optical spectrometer. The spectral resolution and the total spectral channels are $37 \mathrm{kHz}$ and 1.1 million, respectively.

evolution process from interstellar molecules and dust to icy planetesimals or cometary cores and then to planets.

As also reported at this symposium, the space-borne telescopes like ISO and SWAS are adding more and more exciting data. Water is abundant everywhere in the universe, so we need not worry about the existence of oceans on the earthlike planets. Oxygen molecules seem to be much less abundant, according to the report. But again we do not need to be anxious about not having oxygen to breath. It is now believed that the oxygen molecules were not required for the formation and evolution of life in the ocean on the young-phase earth. Oxygen breathing is regarded as a characteristic of evolved stage of life. Moreover, comets might be good carriers of interstellar matter including the various organic molecules to the planetary surfaces.

The subtitle of this symposium is "From Molecular Clouds to Planetary Systems." For me, it seems that the dream by Snyder and Buhl 30 years ago is already almost half realized. What is the remaining half of their (and our) dream?

We have to observe the planets surrounding the other stars directly, find the earth-like planets among them, observe the atmosphere and surface of the 


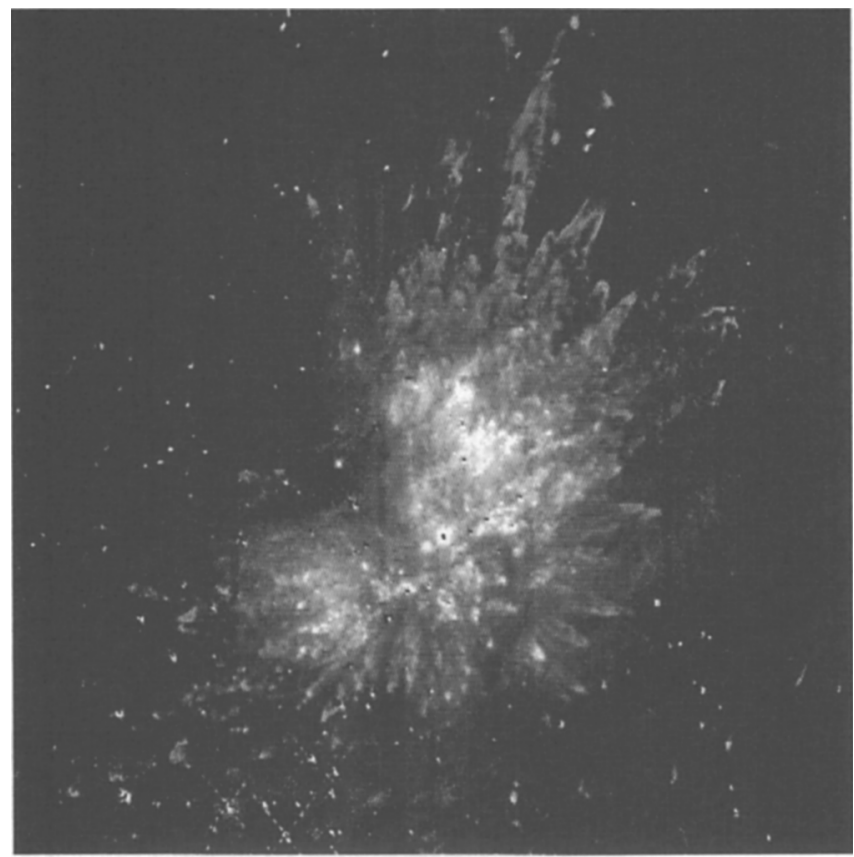

Figure 2. A 2- $\mu \mathrm{m}$ molecular hydrogen image of Orion-IRc2, taken with the Subaru telescope during its first-light in January 1999. This 0.3 arcmin resolution image reveals detailed structure with many shock waves which looks like a "sea urchin," formed by the energetic stellar wind from central massive protostar.

earth-like planets, and then confirm the existence of life on them. A new dream will start then, to understand those lives on each planet and compare those lives with each other. I want to emphasize here that such scientific activities, if realized, will deeply affect our thoughts about life itself on earth. It is a dream to understand our place in the universe.

Will all of this dream be realized in the future? I believe that the answer is "yes," because the progress in observing power is still moving forward, even being in acceleration. I brought here some materials from the Subaru Telescope and its instruments. Let me talk about the new possibilities of observations now and in the future by showing them to you. At the Symposium some initial observational results from the VLT were reported. Subaru is an 8.2-m diameter IR and optical telescope, one of the family of new generation ground-based telescopes, including VLT and GEMINI. The first-light data of the Subaru telescope obtained January 1999 shows very sharp images. One example I show here is part of an image of Orion-KL taken in the $2 \mu \mathrm{m}$ spectral line of the hydrogen molecule. This 0.3 arcsec image shows rich detailed structure of a rapidly expanding gaseous shell (a "sea urchin" - not a "butterfly" as said before!), demonstrating the 
achievement of high spatial resolution. The resolving power of astronomical observations has been increasing very rapidly. For example, in the infrared wavelength region from 1 arcmin of 30 years ago to 1 arcsec of recent days, and it will go to 0.05 arcsec using new generation telescopes with adaptive optics (AO). Subaru released the independent spectra of Pluto and Charon last year. There is much discussion on the spectral assignment of detected features to ethane bands, but what I want to stress here is how quickly we can make progress using the new generation 8-10 m telescopes. The recent Subaru data show a 0.2 arcsec stellar image without $\mathrm{AO}$. We are quite happy with the telescope, and thanks to the steady atmosphere on the summit of Mauna Kea, we will make good use of our AO capabilities.

The new generation telescopes such as VLT, Subaru and GEMINI are very powerful tools for IR observation in astrochemistry. For example, to observe near infrared absorption lines such as $\mathrm{H}_{3}^{+}$or molecular spectra in circumstellar disks, the 0.06 arcsec resolution achieved by Subaru's $8.2 \mathrm{~m}$ aperture combined with the echelle spectrometer of IRCS and AO, will achieve a sensitivity almost 100 times greater than previous observations with conventional 3-4 m class telescopes. The 0.2 arcsec imaging and spectroscopy using COMICS will also provide a big jump for observations in the 10 and $20 \mu \mathrm{m}$ mid IR bands. CIAO, a coronagraphic imager with $\mathrm{AO}$, aims to directly image the planets in extrasolar planetary systems. The huge array of $\sim 100$ millimeter telescopes on the Atacama plateau in Chile, being advanced as the US-Europe plus Japanese collaboration, will start to show its tremendous observational power within 10 years. All of those new tools of astronomy, with some 100 times higher observing capabilities compared with those previously available, will add considerable steps toward the realization of the "latter half" of Snyder and Buhl's dream.

\section{Observations of Extra-Solar-System Life}

We can see further developments coming in the near future. Within 10 to 20 years from now the next generation space telescope (NGST) being discussed in the USA and/or a large space interferometer mission such as Project DARWIN being planned in Europe will be launched into space. They are expected to provide us with the capability to observe the atmospheric composition of earthtype planets. Such observations will enable us to extensively study those planets with high possibility for the existence of life. It is by no means enough, however. The extra-solar-system planets are distant, and they are so small compared with vast distances in the universe.

Possible moon-based huge interferometers or space-borne interferometers which combine 10 or more Subaru-size telescopes might be realized by the middle of the 21'st century. Such huge interferometers with 10,000 to 100,000 times higher resolution than those of Subaru and HST would provide direct surface images of candidate "planets with life," and then may detect the spectral features of forest belts or other indications of life on their surface, if it exists.

The construction of such huge systems in space might be regarded as "another dream". But it should be noted that the current observing sensitivity is more than 100 thousand times higher compared with those of 30 years ago, the time of Snyder and Buhl's detection of $\mathrm{H}_{2} \mathrm{CO}$. Within the next 10 years, the 
additional sensitivity jump will be a factor of 100 . The further jumps described above are without doubt technologically possible. However, due to the very large size, these space-borne interferometers should be realized as a world-wide cooperative project. As all of us realize, we as humankind need to manage a number of serious difficulties facing us on the earth; war, famine, economy, disease, etc. and etc. I would say, however, that humankind is clever enough. And I wish that the 21'st century will be a century when we humankind "meet" other lives in the universe, by the way of astronomical observations, rather than by means of "star ships" which seem very far in the future due to tremendous technological difficulties.

Therefore, I expect that we will meet again at an IAU symposium "From Interstellar Clouds to Life on Various Planetary Systems" sometime in the future.

Thank you very much for your attention.

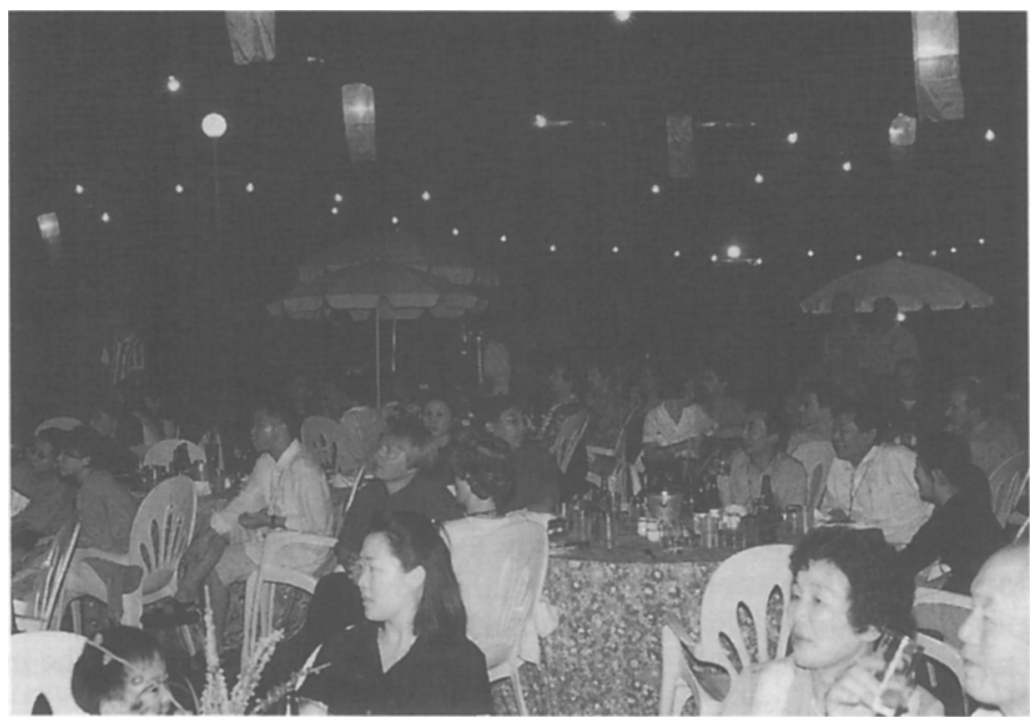

